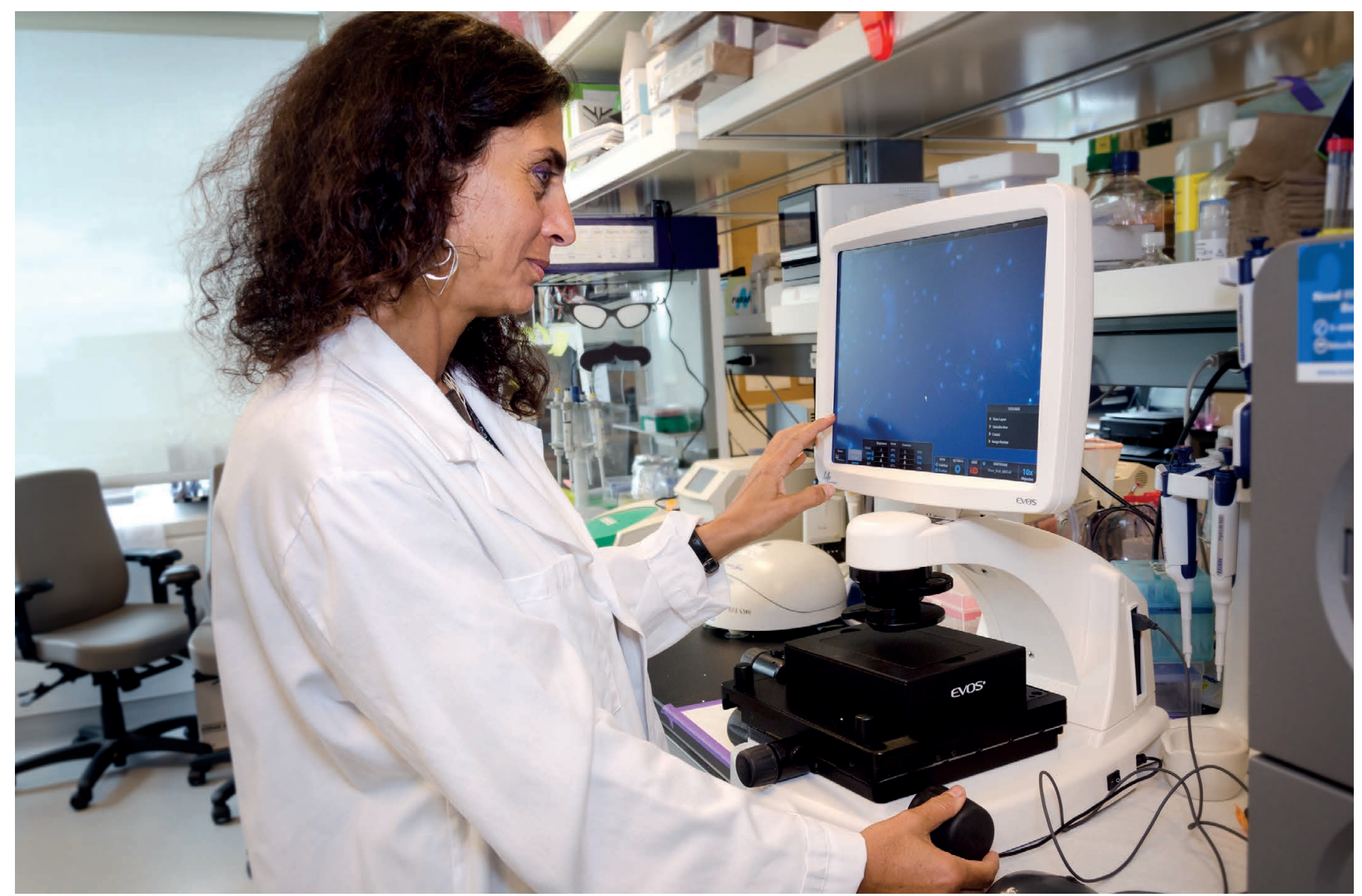

Nada Jabado's research focuses on aggressive brain tumours in children.

\title{
PAEDIATRICS
}

\section{Coming of age}

\section{Brain tumours in children are now known to differ fundamentally from those in adults.}

\section{BY LIAM DREW}

U nder a microscope, gliomas from children and adults are indistinguishable. The cells of such brain tumours resemble glia, the non-neuronal cells of the brain. And in the most aggressive type of these tumours, known as high-grade glioma (HGG), the same telltale signs of rapid proliferation are observed in the young and the old, alike. Tumours in the brains of both groups are nourished by networks of blood vessels and peppered with pockets of cell death.

They show similarities in clinical outcome, too - regardless of a person's age at the time of diagnosis, survival rates are extremely poor. For decades, researchers and doctors therefore thought that children who developed HGG were simply the unluckiest of those affected by an aggressive disease that most often occurred in adults - and, consequently, that any insight gained from the study of such tumours in adults would apply equally to those encountered in children. Nada Jabado, a paediatric oncologist and researcher at McGill University in Montreal, Canada, recalls that in the early 2000s, the treatment for HGG in childhood was identical to that for HGG in an adult. "It was copy and paste," she says.

In adults, these treatment regimens achieved modest improvements in survival times, but they made no difference in children. Indeed, there has been no improvement in paediatric outcomes for more than 30 years.

Faced with this discrepancy, Jabado and a handful of other researchers began to consider that HGG in children might differ fundamentally from that in adults. Initial work indicated that this idea was correct, but no one predicted that the field of paediatric neuro-oncology would be transformed by the discovery, in 2012, of a type of mutation in childhood HGG that had never been seen in adult HGG or in any other form of cancer. "It was a complete shock," says Jabado. The mutations established childhood HGG as a disease in its own right. But even more interestingly, the mutations were in genes that encode histones - the spool-like proteins around which DNA is wound, which regulate gene expression and lie at the centre of the burgeoning field of epigenetics. Suddenly, epigenetics researchers had a disease to study. Histone mutations have since been found in a small number of young-adult HGG subtypes and other rare cancers.

Oncologists are now working to develop treatments that are tailored to the unique biology of HGG in children. "It's really changed out of sight in the last ten years," says Chris Jones, a molecular biologist at the Institute of Cancer Research in London. Research on childhood HGG, he says, "was regarded as a bit of a backwater because nobody knew the biology, and there were no models in which to study it". Numerous groups of researchers are aiming to understand exactly how histone mutations contribute to childhood HGG, in the hope that survival rates can finally be improved.

\section{BACKWATER TO DISCOVERY}

The high-throughput genetic technologies that emerged in the early 2000s revolutionized oncology by enabling researchers to quickly 
acquire large amounts of data from tumour samples. The first generation of such platforms either profiled the expression levels of thousands of genes - which helped to uncover the signalling pathways that drive the uncontrolled division of cancer cells - or scanned the entire genome for large, structural mutations that might be involved in transforming normal cells into cancerous ones. As researchers began to wonder whether deep biological differences might underpin the failure in children of drugs that were designed to treat adult HGG, investigating childhood HGG in this way was an obvious route to follow. But it was not an easy task.

Thankfully, childhood HGG is rare: about eight children in every million develop the disease each year. However, that means there is only a small pool of people from whom researchers can collect samples. The problem is compounded by the fact that, owing to the diffuse and infiltrative nature of childhood HGG tumours, most are inoperable, meaning that few resectioned tumours are available for analysis. Furthermore, by the early 2000s, biopsies of HGG tumours had fallen out of favour because they could bring no clinical benefits, and performing them carried considerable risks.

When Jabado embarked on the first large-scale genetic screen of childhood HGG tumours in 2003, she wrote to more than 300 cancer centres worldwide to request samples. From the seven centres that agreed to help, Jabado and her colleagues managed to accrue 32 usable samples, which was enough to identify two subtypes of childhood HGG - one in which a well-known oncogenic signalling pathway is activated, and another in which it is not - as well as to suggest key differences from adult $\mathrm{HGG}^{1}$.

Suzanne Baker, a developmental neurobiologist at St Jude Children's Research Hospital in Memphis, Tennessee, who began to investigate childhood HGG around the same time as Jabado, gives credit to patient-support groups for explaining to parents of children with the disease that only by allowing biopsies or rapid post-mortem examinations could future patients be helped. She also says that the same groups were instrumental in funding her initial, exploratory studies.

These investigations, conducted with Jones and published in 2010, found that gene-expression changes and large structural mutations that are common in childhood HGG are seen only infrequently in adult HGG, and that genetic signatures that typify adult tumours are rare in paediatric cases ${ }^{2}$. "Most of what we were identifying you could find in both," says Baker, "but the frequency of particular changes was different between adults and kids."

Collectively, the studies by Jabado and Baker established that childhood HGG is diverse and differs from the adult equivalent in interesting ways. In turn, this ensured that when more advanced genetic-sequencing technologies became available that could sequence the whole genome or all genes being expressed

(the exome), and that were capable of identifying mutations at the single-nucleotide level, they were rapidly applied to childhood HGG.

Working independently, Jabado sequenced the exomes of 48 childhood HGG tumours that had developed above the brainstem, and Baker - in collaboration with researchers at Washington University School of Medicine in St Louis - sequenced the entire genomes of 7 brainstem childhood HGG tumours. Baker then explored her team's initial findings in 79 other tumours from inside or above the brainstem. What these groups observed, and published in parallel in January 2012, was the same: mutations in genes encoding histones that had never been seen before in cancers ${ }^{3,4}$. "No one, up until that point," says Jones, "believed that it would be possible for a cell to divide with a mutation in a gene that was encoding something so fundamental as a histone." As scaffolds for genomic DNA, histones are highly conserved proteins that help to shape the $3 \mathrm{D}$ arrangement of the genome, through which they influence the expression of genes. Consequently, most biologists had thought that any mutations in histones would disrupt gene regulation considerably and thereby impair cell survival. Yet cancer cells do not struggle to survive; they are defined by their relentless division and an unwillingness to die.

So it was even more remarkable that Jabado, Baker and other researchers soon confirmed that there was not just one histone mutation but three. The most common one affects a specific amino acid in histone $\mathrm{H} 3.3$ and is found in about two-thirds of brainstem childhood HGG tumours, as well as in other tumours that form along the midline of the central nervous system. Another mutation - in the gene that encodes $\mathrm{H} 3.1$, but that affects

"The frequency of particular changes was different between adults and kids." the same amino acid as in H3.3 - occurs in brainstem tumours in very young children. And tumours of the cerebral cortex contain the third mutation, which affects a different amino acid in H3.3. No individual childhood HGG has yet been shown to contain more than one type of histone mutation. Overall, histone mutations account for slightly more than half of all childhood HGG cases.

Researchers also found, however, that a histone mutation was never the sole mutation in such tumours. Other mutations, typically in well known oncogenes, were always present. Strikingly, there seemed to be preferred partnerships between mutations - an idea that was confirmed by an ever increasing number of samples, and that culminated in a survey of the genomes of 1,067 childhood HGG tumours, published in 2017, which delineated 10 subgroups, each defined by a distinctive collection of mutations ${ }^{5}$.

These partnerships seem to form early in tumorigenesis and, according to observations by Jabado's group and others, remain stable throughout progression of the disease. Stefan Pfister, a paediatric neuro-oncologist at the National Center for Tumor Diseases in Heidelberg, Germany, who collaborates with Jabado, thinks that the genomes of childhood HGG tumours that are defined by pairs of mutations are more straightforward than those of adult HGG tumours, which contain combinations of many acquired mutations. This comparative simplicity makes these tumours "better candidates for successful therapeutic intervention", Pfister says.

Mutations in subtypes of childhood HGG known to be involved in oncogenic pathways in other cancers provide promising leads for using previously developed therapeutic agents to treat children who have specific types of HGG. Initial trials have produced encouraging results in two subtypes that do not contain histone mutations. But neuro-oncologists remain desperate to understand fully the biology that underlies histone mutations, in the hope that these mutations will be an Achilles heel that opens the door to innovative treatments.

\section{THE HISTONE EXPLANATION}

Histone biology is complicated. Each of the five families of histones comprises multiple variants that are encoded by multiple genes. And histones are not merely an inert scaffold for DNA. Rather, through the addition or removal of chemical moieties such as methyl or acetyl groups at specific amino acids, histones are able to alter the conformation of nearby DNA to allow or prevent the transcription of genes.

Often, the modified amino acid that controls this change in conformation is lysine. The two mutations found in brainstem and midline childhood HGG tumours that affect the same amino acid in the closely related histones $\mathrm{H} 3.1$ and $\mathrm{H} 3.3$ replace a crucial lysine at position 27 in these proteins with the amino acid methionine. Oncologists refer to these mutated histones as oncohistones, and use the shorthand $\mathrm{K} 27 \mathrm{M}$ (in which lysine is $\mathrm{K}$, and methionine is $\mathrm{M}$ ) to describe the mutation.

Whereas the lysine is modified chemically - by the addition or removal of a methyl group - to regulate gene expression, the methionine is not. Moreover, the K27M mutation affects not only the histone gene in which it occurs; in fact, the methionine broadly inhibits the enzyme that adds methyl groups to lysines in histones. This effect leads to widespread changes in histone structure and, therefore, to more extensive changes in DNA organization and gene expression.

Much less is understood about the rare third mutation that is found in tumours of the cerebral cortex. It affects a glycine at position 34 in H3.3, but its effects are probably mediated by inhibiting the methylation of the nearby lysine at position 36 . The importance of this lysine is substantiated by the observation that a subtype of childhood HGG of the cerebral cortex that lack this histone mutation have, 


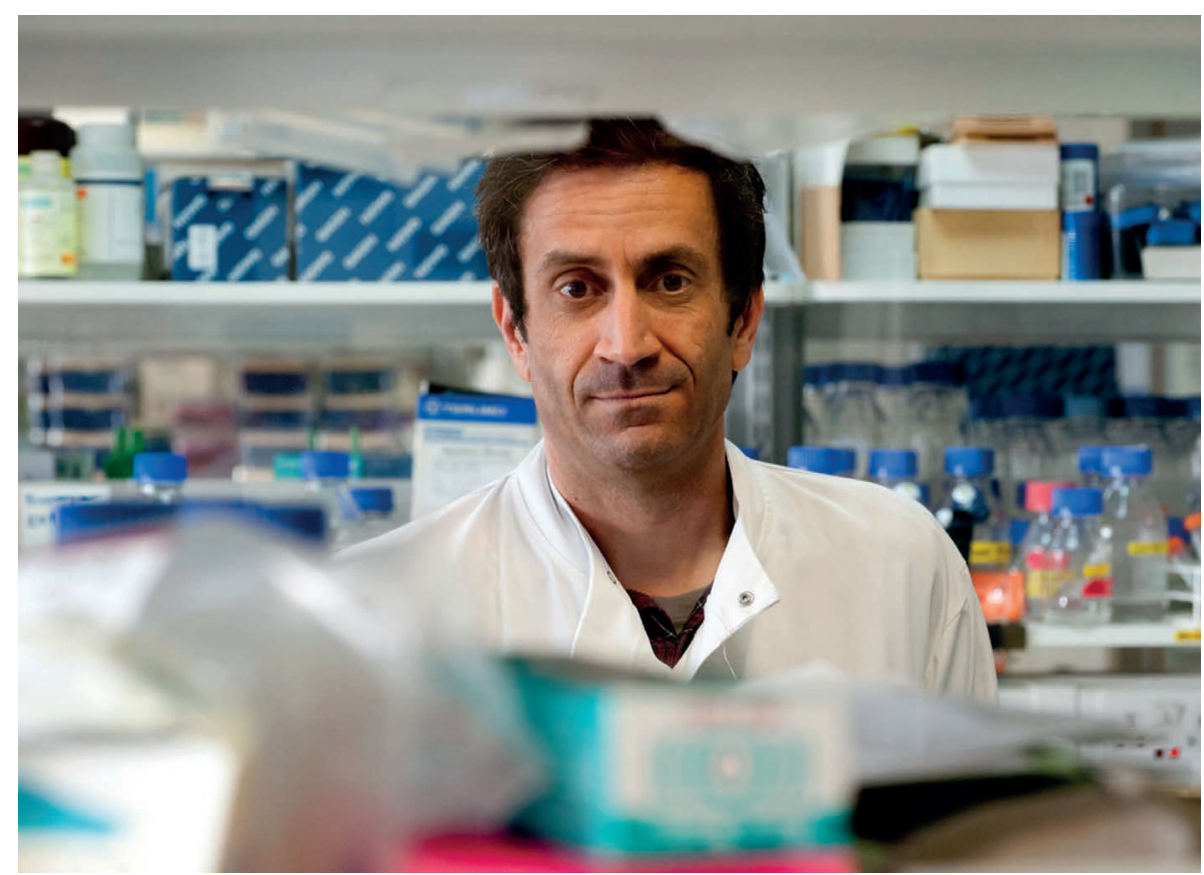

Chris Jones is helping to define how glioma in childhood differs from the disease in adults.

instead, a mutation in the enzyme that adds methyl groups to the lysine.

How these histone mutations might lead a developing brain cell to go rogue is the focus of ongoing research; the more that can be found out about the direct and downstream effects of such mutations, the more potential targets for intervention there will be. In mice, introducing the K27M mutation into embryonic neural progenitor cells together with a mutation in the common oncogene Trp53 - a frequent partner mutation in childhood HGG - induces a tumour to form. But this happens only if the genes are switched on at the correct point in the animal's development. These and other findings suggest that there is a unique and transient set of circumstances in the developing brain that allows a certain type of cell to thrive when it acquires these mutations.

"These are really unusual tumours," says Baker, who suggests that childhood HGG, unlike that in adults, is best understood as a developmental disease. Developing brains are packed with cells that are dividing rapidly, and histone mutations seem to divert those cells along the path towards cancer, or to trap them at a particular developmental stage. Either route places cells in a state that is vulnerable to the effects of partner mutations.

But researchers who hope to use information about histone mutations to help treat HGG in children must address a crucial question. If oncohistones are responsible only for establishing the initial conditions under which such tumours develop, but are not required for the long-term survival of cancer cells, would targeting these histones with drugs after a tumour has been established provide any benefit? For histones to be a suitable target, they must have an ongoing role in tumour-cell survival or proliferation.
From work conducted in cell lines and mouse models, respectively, Baker and Jabado both now think they have compelling evidence that the K27M mutation remains essential for tumour progression. Following removal of the histone mutation, says Jabado, "there are no tumours that form - regardless of what else has happened."

These findings support results from drug screens for histone-modifying compounds that also kill tumour cells. One such study was led by Michelle Monje, a neuro-oncologist at Stanford University

School of Medicine in

California. Monje found that panobinostat - a cancer drug developed by pharmaceutical firm Novartis of Basel, Switzerland, that inhibits enzymes that remove acetyl groups from his-

"There's a lot of optimism now in an area where there was huge pessimism for decades." tones - was effective in killing cells derived from childhood HGG tumours ${ }^{6}$. A phase I clinical trial of panobinostat is scheduled to end in October 2019. Other drugs that target pathways that regulate histones and gene expression have also shown promise in preclinical testing.

Nevertheless, potential treatments need not necessarily target the mutations that cause tumours. Monje, for example, has found that tumour growth might be inhibited by blocking a signalling molecule released by neurons that seems to be important for stimulating cell proliferation in childhood HGG. She has also collaborated on an immunotherapybased approach that uses engineered $T$ cells to target a cell-surface protein that is probably expressed by such tumours as a non-specific consequence of the K27M mutation.
Jabado foresees treatment regimens that take three lines of attack: a drug that directly tackles the epigenetic effects of the histone mutation; a drug that targets the partner mutation's effects; and an immunotherapeutic agent. "It's going to be a matter of combination," she predicts.

As the prospect of treatments for childhood HGG moves closer, the disease's rarity will continue to be an obstacle. Finding participants for trials could be difficult, especially now that childhood HGG is known to comprise 10 subtypes - each demanding its own therapeutic strategy while being about an order of magnitude rarer than is HGG overall. This also has ramifications for attracting the attention of pharmaceutical companies, which might be put off by small market sizes.

Here, patient-support groups are again likely to be crucial, this time in lobbying pharmaceutical companies to get involved in tackling childhood HGG - for example, by supplying drugs for preclinical and clinical research. But Pfister is optimistic that such firms will want to pursue treatments for the disease, citing a successful trial of larotrectinib in a subtype of childhood HGG that lacks oncohistones. The drug, developed by Loxo Oncology, a small biotechnology company in Stamford, Connecticut, was designed to inhibit enzymes that are affected by a class of mutations found in various other cancers. Subsequently, it was considered to be appropriate for treating an HGG subtype on the basis of the glioma's defining mutation.

With regard to finding children to take part in trials, Pfister stresses that because so few are affected, researchers must ensure that only treatment options with high potential are tested. Patients should not be enrolled in trials prematurely by oncologists who are "rushing something into the clinic because we feel under pressure to do something", he says. Jabado thinks that because of the relative simplicity of the biology that underlies each subtype of childhood HGG, small trials will be sufficient. "If it's going to work, we're going to see it," she says.

Once a neglected speciality, paediatric neuro-oncology is now a thriving discipline with a focus on genetic disruptions that have captivated a wider audience. "There's a lot of optimism now in an area where there was huge pessimism for decades," says Jones. Of course, anyone who has interacted with a child with HGG knows that it is not enough just to investigate fascinating biology. "We're all acutely aware that we haven't yet turned this directly into patient benefit," Jones says. "But most of us believe that we're on the cusp." -

Liam Drew is a freelance science writer based in London.

1. Faury, D. et al. J. Clin. Oncol. 25, 1196-1208 (2007).

2. Paugh, B. S. et al. J. Clin. Oncol. 28, 3061-3068 (2010).

3. Wu, G. et al. Nature Genet. 44, 251-253 (2012).

4. Schwartzentruber, J. et al. Nature 482, 226-231 (2012).

5. Mackay, A. et al. Cancer Cell 32, 520-537 (2017)

6. Grasso, C. S. et al. Nature Med. 21, 555-559 (2015). 